\title{
Soluções Superluminais de Energia Finita das Equações de Maxwell
}

E.C. de OLIVEIRA, 1 , Departamento de Matemática Aplicada, Instituto de Matemática, Estatística e Computação Científica, Unicamp, 13083-970 Campinas, SP, Brasil.

W.A. RODRIGUES Jr. ${ }^{2}$, Department of Mathematics, University of Liverpool, Liverpool L69 3BX, UK.

Resumo Obtém-se soluções (exatas) superluminais de energia finita para as equações de Maxwell e discute-se o significado físico de tais soluções.

\section{Introdução}

Recentemente alguns trabalhos $[6,17]$ apareceram na literatura mostrando que em algum meio hipotético existe a possibilidade da existência de pulsos eletromagnéticos superluminais (soluções das equações de Maxwell) tal que suas frentes viajam no meio com velocidades superluminais. As soluções descobertas em [6, 17], apesar de seu interesse teórico têm energia infinita e como tal não podem ser produzidas no mundo físico. Somente aproximações de abertura finita para estas ondas podem eventualmente ser produzidas (supondo a existência do tal meio especial). O objetivo deste trabalho é mostrar que em contraste com as soluções descobertas em $[6,17]$ (que, como já afirmado têm energia infinita), existem soluções das equações de Maxwell no vácuo que são soluções (exatas) superluminais de energia finita. Estas soluções, como veremos, aparecem como soluções de problemas tipo Sommerfeld $[1,9]$. Estudamos também o aparente paradoxo que ocorre quando uma solução superluminal gerada em um sistema inercial $L$ é observada pelos observadores em repouso em um sistema inercial $Z$ que se move com velocidade $|\vec{V}|=V$ em relação a $L$. Discutimos também se tais soluções podem ser geradas no mundo físico.

\section{Pulso Escalar Superluminal}

Começa-se lembrando como escrever configurações do campo eletromagnético em termos dos potenciais de Hertz [15, 11]. Suponha-se que temos um potencial de

\footnotetext{
${ }^{1}$ capelas@ime.unicamp.br

${ }^{2}$ W.Rodrigues@liverpool.ac.uk. Em afastamento do Departamento de Matemática Aplicada, Instituto de Matemática, Estatística e Computação Científica, Unicamp, 13083-970 Campinas, SP.
} 
Hertz, $\vec{\Pi}_{m}$, de tipo magnético. ${ }^{3}$ Então, o campo eletromagnético associado é dado por

$$
\vec{E}=-\frac{\partial}{\partial t}\left(\nabla \times \vec{\Pi}_{m}\right), \quad \vec{B}=\nabla \times \nabla \times \vec{\Pi}_{m}
$$

Toma-se $\vec{\Pi}_{m}=\Phi \hat{e}_{z}$. Logo, desde que o potencial de Hertz (no vácuo) satisfaz a equação de onda homogênea, tem-se que

$$
\square \Phi=0
$$

O problema de Sommerfeld (não deve ser confundido com o problema de Cauchy) a ser considerado aqui é o seguinte: Em um dado sistema de referência (laboratório ${ }^{4}$ ) procuramos por uma solução $\Phi_{X}:(t, \vec{x}) \mapsto C$ (onde $C$ é o corpo dos números complexos) para a equação 2.1 satisfazendo as seguintes condições de contorno ${ }^{5}$ no plano $z=0$,

$$
\left\{\begin{array}{l}
\Phi_{X}(t, \rho, 0)=\mathbf{T}(t) \int_{-\infty}^{\infty} d \omega B(\omega) J_{0}(\omega \rho \operatorname{sen} \eta) e^{-i \omega t} \\
\left.\frac{\partial \Phi_{X}(t, \rho, z)}{\partial z}\right|_{z=0}=i \mathbf{T}(t) \cos \eta \int_{-\infty}^{\infty} d \omega B(\omega) J_{0}(\omega \rho \operatorname{sen} \eta) k(\omega) e^{-i \omega t}
\end{array}\right.
$$

onde $\mathbf{T}(t)=[\Theta(t+T)-\Theta(t-T)], \Theta$ é a função de Heaviside, $k(\omega)=\omega$, e $\eta$ é uma constante chamada ângulo de axicon $[12,2,5,13,3,8]$ e $B(k)$ é uma distribuição de freqüências apropriada. Como mostrado em [2] a solução da equação 2.1 (para $z>0, t>T)$ que satisfaz as condições de Sommerfeld é

$$
\Phi_{X}(t, \rho, z)=\left\{\begin{array}{ccc}
\int_{-\infty}^{\infty} d \omega B(\omega) J_{0}(\omega \rho \operatorname{sen} \eta) e^{-i \omega(t-z \cos \eta)} & \text { para } & |t-z \cos \eta|<T \\
0 & \text { para } & |t-z \cos \eta|>T
\end{array}\right.
$$

Chamamos $\Phi_{X}$ de um pulso escalar $X$ superluminal. Agora, como é bem conhecido, a densidade de energia para uma configuração do campo complexo, como $\Phi_{X}$, é

$$
u=\left(\partial_{t} \Phi_{X}\right)\left(\partial_{t} \Phi_{X}^{*}\right)+\left(\partial_{x} \Phi_{X}\right)\left(\partial_{x} \Phi_{X}^{*}\right)+\left(\partial_{y} \Phi_{X}\right)\left(\partial_{y} \Phi_{X}^{*}\right)+\left(\partial_{z} \Phi_{X}\right)\left(\partial_{z} \Phi_{X}^{*}\right),
$$

e a energia da configuração do campo pode ser calculada pela integral de volume de $u$ num hiperplano de tempo constante, digamos $t=T^{\prime}>T$. O cálculo se torna mais simples se efetuado em coordenadas cilíndricas. Lembrando que da equação 2.3 segue-se que o suporte do pulso em $t=T^{\prime}$ é $\triangle z=2 T / \cos \eta$, temos

\footnotetext{
${ }^{3}$ No que se segue usam-se unidades tais que a velocidade da luz seja $c=1$.

${ }^{4} \mathrm{O}$ laboratório é modelado por um campo vetorial tipo tempo $\partial / \partial t$.

${ }^{5} \mathrm{~A}$ necessidade destas condições de contorno é provada em [12].
} 


$$
\mathcal{E}=\frac{8 \pi T}{\operatorname{sen}^{2} \eta \cos \eta} \int_{-\infty}^{\infty}|B(k)|^{2} k d k,
$$

onde os termos de energia cinética e potencial têm contribuições iguais. A equação 2.4 fornece energia finita para o pulso escalar $X$ para uma infinidade de funções distribuições de freqüência $B(k)$, tais que $|B(k)|^{2}$ seja nulo para $k<0$. Um exemplo trivial é $B(k)=\left[\Theta(k)-\Theta\left(k-k_{0}\right)\right]$, com $k_{0}$ uma constante.

\section{Caso Eletromagnético}

Agora, estuda-se o caso eletromagnético. As componentes não nulas do campo eletromagnético ${ }^{6}$ correspondendo a um potencial de Hertz magnético $\vec{\Pi}_{m}=\Phi_{X} \hat{e}_{z}$ é $($ para $z>0, t>T)$

$$
\begin{cases}E_{\theta}=i \operatorname{sen} \eta \int_{-\infty}^{\infty} d k B(k) k^{2} J_{1}(k \rho \operatorname{sen} \eta) e^{-i k(t-z \cos \eta),} & \\ B_{\rho}=\frac{-i}{2} \operatorname{sen} 2 \eta \int_{-\infty}^{\infty} d k B(k) k^{2} J_{1}(k \rho \operatorname{sen} \eta) e^{-i k(t-z \cos \eta),} & \text { para }|t-z \cos \eta|<T, \\ B_{z}=\operatorname{sen}^{2} \eta \int_{-\infty}^{\infty} d k B(k) k^{2} J_{0}(k \rho \operatorname{sen} \eta) e^{-i k(t-z \cos \eta)}, & \\ E_{\theta}=B_{\rho}=B_{z}=0, & \text { para }|t-z \cos \eta|>T .\end{cases}
$$

Agora, usando a densidade de energia padrão do campo elétrico [15, 11], a energia do pulso eletromagnético $X$ superluminal resulta em,

$$
\begin{aligned}
\mathcal{E}_{X} & =\frac{1}{2} \int_{0}^{2 \pi} \int_{z_{\min }}^{z_{\max }} \int_{0}^{\infty}\left[E_{\theta} E_{\theta}^{*}+B_{\rho} B_{\rho}^{*}+B_{z} B_{z}^{*}\right] \rho d \rho d z d \theta \\
& =\frac{4 \pi T}{\cos \eta} \int_{-\infty}^{\infty}|B(k)|^{2} k^{3} d k .
\end{aligned}
$$

A equação 3.1 fornece energia finita para soluções superluminais das equações de Maxwell satisfazendo as condições de contorno de Sommerfeld (aqui expressas através das condições para o potencial de Hertz associado) para uma infinidade de possíveis distribuições de freqüências $B(k)$, como no caso escalar.

\section{Conclusões}

Temos quatro comentários a fazer antes de terminar este trabalho, a saber:

${ }^{6}$ Chamado de um pulso eletromagnético $X$ superluminal $[12,2]$. 
(i) Qual é a nossa solução de energia finita (para a equação de onda escalar) como vista por um observador num sistema de Lorentz $Z \in \sec T M$,

$$
Z=\frac{1}{\sqrt{1-V^{2}}}\left(\partial_{t}+V \partial_{z}\right)
$$

que está em movimento com velocidade $V=\cos \eta$ relativa ao laboratório (o sistema $\left.L=\partial_{t} \in \sec T M\right) ?$

Como pode ser verificado facilmente a solução transformada é:

$$
\Phi_{X}^{\prime}\left(t^{\prime}, \rho, z^{\prime}\right)=\left\{\begin{array}{cll}
\int_{-\infty}^{\infty} d \omega B(\omega) J_{0}(\omega \rho \operatorname{sen} \eta) e^{-i \omega \operatorname{sen} \eta t^{\prime}}, & \text { para } & \left|t^{\prime}\right|<T / \operatorname{sen} \eta \\
0, & \text { para } & \left|t^{\prime}\right|>T / \operatorname{sen} \eta .
\end{array}\right.
$$

A solução é independente da coordenada espacial z e corresponde a uma onda estacionária ocupando "espaço de repouso" do sistema $Z$ e que existe somente para o intervalo de tempo $\triangle t^{\prime}=2 T / \operatorname{sen} \eta$. É este um resultado não físico? Se não, qual é o significado de tal onda para os observadores do sistema $Z$ ? Com um diagrama de Minkowski pode-se mostrar que a onda é estacionária por um período finito de tempo e depois desaparece, de acordo com a ordenação do tempo do sistema $Z$, porque ela está indo o para passado dos observadores $Z$. Isto deve ser um fenômeno normal se a teoria da relatividade é verdadeira, e movimentos superluminais genuínos existem. Os observadores no sistema $Z$ computarão uma energia infinita para aquela onda, mas desde que eles conheçam a teoria da relatividade eles interpretarão o fenômeno como segue: a onda estacionária existente, por um período finito de tempo, em nosso sistema de referência é uma onda superluminal de energia finita produzida no laboratório (o sistema $L$ ) que se move com velocidade $-1 / \cos \eta$ relativamente ao nosso sistema (i.e., o sistema $Z$ ). Naturalmente, os físicos do sistema $Z$ não podem produzir tal onda em seu sistema, devido a duas razões. A primeira razão é que a onda, de acordo com eles, tem energia infinita e a segunda razão, que é também a crucial é que o dispositivo que a produz está em repouso em um outro sistema (o sistema $L$ ). De acordo com o Princípio de Relatividade os físicos do sistema $Z$ podem reproduzir em seus sistemas o dispositivo usado no sistema $L$ e lançar uma onda como aquela dada pela equação 2.3 (com condições de contorno do tipo da equação 2.2) com $(t, \rho, z)$ substituídos por $\left(t^{\prime}, \rho, z^{\prime}\right)$. Naturalmente, se isso fosse possível chegaríamos a situações paradoxais bem conhecidas $^{7}$, que fortunadamente não necessitam ser discutidos aqui (veja (iii) abaixo).

Note também que os matemáticos que habitam o sistema $Z$ concientes da interpretação de seus colegas físicos podem obter diretamente a solução dada pela equação 4.1, resolvendo um problema de valor no contorno misto, onde as condições de contorno são:

$$
\left.\Phi_{X}^{\prime}\left(t^{\prime}, \rho, z^{\prime}\right)\right|_{z^{\prime}=-\cos \eta t^{\prime}}=\mathbf{T}\left(t^{\prime}, T, \eta\right) \Omega\left(\rho, \eta, \omega, t^{\prime}\right)
$$

\footnotetext{
${ }^{7}$ Mais detalhes são encontrados em [8].
} 
e

$$
\left.\left(\gamma \frac{\partial}{\partial z^{\prime}}-\gamma V \frac{\partial}{\partial t^{\prime}}\right) \Phi_{X}^{\prime}\left(t^{\prime}, \rho, z^{\prime}\right)\right|_{z^{\prime}=-\cos \eta t^{\prime}}=i \cos \eta \mathbf{T}\left(t^{\prime}, T, \eta\right) \Omega\left(\rho, \eta, \omega, t^{\prime}\right),
$$

onde introduzimos a notação $\mathbf{T}\left(t^{\prime}, T, \eta\right)=\left[\Theta\left(\operatorname{sen} \eta t^{\prime}+T\right)-\Theta\left(\operatorname{sen} \eta t^{\prime}-T\right)\right]$ e

$$
\Omega\left(\rho, \eta, \omega, t^{\prime}\right)=\int_{-\infty}^{\infty} d \omega B(\omega) J_{0}(\omega \rho \operatorname{sen} \eta) e^{-i \omega \operatorname{sen} \eta t^{\prime}}
$$

(ii) Naturalmente, uma análise análoga vale para as soluções superluminais de energia finita das equações de Maxwell que encontramos. Observamos aqui que a existência das soluções superluminais encontradas, não estão em conflito com o famoso resultado do problema de Cauchy concernente às equações de Maxwell. Aquele resultado afirma: "qualquer configuração do campo eletromagnético com suporte compacto em $t=0$, digamos para $|\vec{x}| \leq R$ é tal que o campo é nulo para $t>0$ para todo $|\vec{x}| \geq R+t "{ }^{8}$ De fato, as soluções encontradas como solução de nosso problema de Sommerfeld são tais que para qualquer $t>T$ a onda e suas derivadas normais não possuem suporte compacto em nenhuma hipersuperfície tipo espaço, $t=T^{\prime}, T^{\prime}>T$.

(iii) É possível construir um dispositivo físico de modo a lançar um pulso eletromagnético superluminal $X$ com energia finita? Nossa resposta é não. Realmente, aproximações de abertura finita (AAF) para a produção experimental de soluções superluminais tipo $X$ das equações de Maxwell (que, naturalmente têm energia finita) já foram obtidas [14, 10], como previstas em [13, 3, 8]. Entretanto, estas AAF são tais que seus picos movem-se [12] com velocidade $v>1$ mas suas frentes sempre se movem com a velocidade da luz. Este resultado foi previsto em [8] e endossado por resultados experimentais de [10] como provado em [12]. Agora, no que concerne as soluções que encontramos, a tentativa de produzi-las (por uma antena) como ondas físicas reais implicaria na existência de uma antena que se estendesse em todo plano $z=0$, durante o intervalo de tempo $-T<t<T$. Naturalmente, isto é fisicamente impossível pois não existem antenas de área infinita.

(iv) Além das soluções superluminais encontradas, existem também soluções $s u b$ luminais de energia finita (que serão discutidas oportunamente). Ainda que as novas soluções superluminais não possam ser produzidas por dispositivos físicos, acreditamos que uma possível razão para a não existência natural destas 'partículas' de luz em nosso universo, é que se elas existissem implicariam imediatamente em uma violação do Princípio de Relatividade. Conjecturamos ainda que a existência destas novas soluções podem eventualmente também encontrar aplicações no entendimento de alguns problemas fundamentais concernentes ao fenômeno da não localidade em mecânica quântica [7], mas não discutiremos tais questões nesta breve nota.

Agradecimentos: Agradecemos os Drs. D.S. Thober and A.L. Xavier por várias discussões e ao Dr. I. Porteous por ter lido atentamente o manuscrito.

\footnotetext{
${ }^{8}$ Uma prova de um teorema análogo para a equação de onda homogênea pode ser encontrado em [16]. Para as equações de Maxwell veja [4].
} 
Abstract: We exhibit exact finite energy superluminal solutions of Maxwell equations in vacuum and discuss the physical meaning of these solutions.

\section{Referências}

[1] L. Brillouin, "Wave Propagation and Group Velocity", Academic Press, New York, 1960.

[2] E. Capelas de Oliveira, W.A. Rodrigues Jr, D.S. Thober and A.L. Xavier, Thoughtful comments on 'Bessel beams and signal propagation', Phys. Lett. A, 284 (2001), 296-303.

[3] E. Capelas Oliveira and W.A. Rodrigues, Jr., Superluminal electromagnetic waves in free space, Ann. der Physik, 7 (1998), 654-659.

[4] R. Courant and D. Hilbert, "Methods of Mathematical Physics", vol. 2, John Wiley and Sons, New York, 1966.

[5] J. Durnin, Exact solutions for nondiffracting beams. I. The scalar theory, J. Opt. Soc. Am. A, 4 (1987), 651-654.

[6] P. Ghose and M.K. Samal, Lorentz invariant superluminal tunneling, Phys. Rev. E, 64 (2001), to appear.

[7] A.A. Grib and W.A. Rodrigues, Jr., "Nonlocality in Quantum Physics", Kluwer Acad./Plenum Publ., New York, 1999.

[8] J.E. Maiorino and W.A. Rodrigues, Jr., What is Superluminal Wave Motion?, electronic book at http://www.cptec.br/stm, Sci. and Tech. Mag., 4, No. 2 (1999).

[9] F.A. Mehmeti, "Transient Tunnel Effect and Sommerfeld Problem", Akademie Verlag, Berlin, 1996.

[10] D. Mugnai, A. Ranfagni and R. Ruggeri, Observation of superluminal behaviors in wave propagation, Phys. Rev. Lett., 84 (2000), 4830-4833.

[11] W.K.H. Panofski and M. Phillips, "Classical Electricity and Magnetism", 2nd ed., Addison-Wesley, Reading, MA, 1962.

[12] W.A. Rodrigues Jr., D.S. Thober and A.L. Xavier, Jr., Causal explanation for observed superluminal behavior of microwave propagation in free space, Phys. Lett. A, 284 (2001), 217-224.

[13] W. A. Rodrigues, Jr. and J.Y. Lu, On the existence of undistorted progressive waves (UPWs) of arbitrary speeds $0 \leq v \leq \infty$ in nature, Found. Phys., 27 (1997), 435-508. 
[14] P. Saari and K. Reivelt, Evidence of $X$-shaped propagation-invariant localized light waves, Phys. Rev. Lett., 21 (1997), 4135-4138.

[15] J.A. Stratton, "Electromagnetic Theory", McGraw-Hill, New York, 1941.

[16] M.E. Taylor, "Pseudo Differential Operators", Princeton Univ. Press, Princeton, 1981.

[17] X. Zhou, Possibility of a light pulse with speed greater than $c$, Phys. Lett. A, 278 (2001), 1-5. 
